\section{THU0037 IMMUNOGENICITY IN A TERTIARY CARE HOSPITAL: OUR EXPERIENCE}

A.G. Valle ${ }^{1}$, M. Garijo Buffort ${ }^{2}$, M. Retuerto Guerrero ${ }^{2}$, C. Iñiguez ${ }^{2}$, E. Díez ${ }^{2}$, C. Moriano ${ }^{2}$, A. López ${ }^{2}$, M. Martín ${ }^{2}$, C. Álvarez ${ }^{2}$, T. Pérez Sandoval ${ }^{2}$.

${ }^{1}$ Rheumathology, Hospital Can Misses, Ibiza; ${ }^{2}$ Rheumathology, Complejo

Asistencial Universitario, León, Spain

Background: The drugs called Anti-TNF inhibitors are capable of inducing an immune response (immunogenicity) Its effectiveness may be affected by the development of Anti-Drug Antibodies (Ab)

Objectives:

- To assess the frequency of appearance of Anti-Drug Antibodies: Infliximab (IFX), Adalimumab (ADA), etanercept (ETN)

- To classify the failures of response

- To analyse the relationship between anti-TNF $\alpha$ antibodies and concomitant treatment with DMARDs

- To observe whether there is a link between risk factors and drug levels

Methods: This is a retrospective, descriptive, observational study of patients with Rheumatoid Arthritis (RA), Spondyloarthritis ( $S p A)$, Psoriatic arthritis (PsA), Seronegative arthritis (SA) and Enteropathic arthritis (EA) with active disease and that were treated in the "University Health Care Hospital of León" between Jan-2015 and Jan-2016. Using ELISA Technology and the kits Promonitor ${ }^{\circledR}$, it was possible to detect serum levels of IFX ADA, ETN (reference values $>2.5 \mu \mathrm{g} / \mathrm{mL}$, $5-8 \mu \mathrm{g} / \mathrm{mL}$ and $0.8-1.2 \mu \mathrm{g} / \mathrm{mL}$ respectively) and of anti-drug antibodies. The samples were collected the same day of the administration, prior to it, always in a trough level. The gathered data was: demographic data, activity, time-to-disease progression, prior treatment with biologics, concomitant DMARDs, duration of the biologic treatment and dosage, quantization levels, anti-TNF antibodies, cardiovascular risk factors (CVRF) and smoking habits.

Results: Variables to study:

$\mathrm{N}=40$ : IFX 50\%, ADA 30\%, ETN $20 \%$.

Age $53.6 \pm 3.7$ years old $[95 \% \mathrm{Cl}$, Women: $55 \%$, time-disease progression: $12.3 \pm 2.7$ years old.

Type of disease: RA $47.5 \%$, SpA $15 \%$, PsA $20 \%$, SA $7.5 \%$, EA $10 \%$.

DAS28: $3.5 \pm 0.4$, BASDAI $4.7 \pm 0.5$, BASFI $4.3 \pm 1.4$

Prior treatment with biologics (30\%): IFX 15\%, ADA 66\%, ETN $12.5 \%$.

Frequency of administration: IFX $8.6 \pm 0.36$ weeks, ADA $2.25 \pm 0.36$ weeks, ETN

$1.1 \pm 1.0$ weeks.

Reasons for requesting the levels:

- Secondary failure (82\%): IFX 90\%, ADA $66.7 \%$, ETN $75 \%$

- Primary failure (17\%): IFX 5\%, ADA 33\%, ETN $25 \%$

- Infusion reactions (2.5\%)

Drug levels within the therapeutic range: IFX 10\%, ADA 41\%, ETN 50\%.

Formation of anti-TNF Ab of the sample: IFX $30 \%$, ADA 16\%, ETN $0 \%$.

DMARDs: presence of $62.5 \%$ (MTXsc $64 \%$, MTXvo $20 \%$, Leflunomide, Sul-

fasalazine e Hydroxychloroquine $16 \%$ ).

Conclusions: We found the following conclusions:

- In the data collected, we observe that the IFX (30\%) is the most immunogenic drug, followed by the ADA $(16 \%)$ and being the ETN $(0 \%)$ the one that so far has not presented anti-drug Ab, outcomes in agreement with the medical literature

- The main reason for requesting has been the secondary failure (90\%)

- The suboptimal levels of the drug and the presence of specific ab are correlated with the loss of clinical response. In our case, the proper range of drug is only objective in $10 \%$ of the patients treated with IFX, $41 \%$ with ADA and $50 \%$ with ETN

- The concomitant use of DMARDs in our study has not been shown to decrease levels of Ab, being the MTX the most used in our patients (84\%). We observed no correlation between the occurrence of $A b$, the use of DMARDs or the type of disease

- The monitoring of the levels of anti-TNF drug may be useful to individualize the treatment, to avoid possible side effects and to make decisions regarding the continuation or change of therapy.

Disclosure of Interest: None declared

DOI: 10.1136/annrheumdis-2017-eular.2800

\section{THU0038 BIMEKIZUMAB DUAL INHIBITION OF IL-17A AND IL-17F PROVIDES EVIDENCE OF IL-17F CONTRIBUTION TO CHRONIC INFLAMMATION IN DISEASE-RELEVANT CELLS}

A. Maroof ${ }^{1}$, R. Okoye ${ }^{1}$, T. Smallie ${ }^{1}$, D. Baeten ${ }^{2}$, S. Archer ${ }^{1}$, C. Simpson ${ }^{1}$, M. Griffiths ${ }^{1}$, S. Shaw ${ }^{1} .{ }^{1}$ UCB Pharma, Slough, United Kingdom; ${ }^{2}$ UCB Pharma, Brussels, Belgium

Background: IL-17A and IL-17F share structural homology and have similar biological function ${ }^{1}$. Although the contribution of IL-17A to immune-mediated inflammatory diseases has been widely reported ${ }^{1-3}$, the role of IL-17F is less well characterised in human tissue inflammation. Bimekizumab, a humanised monoclonal IgG1 antibody, was developed to neutralise both IL-17A and IL-17F potently and selectively, and is under clinical development as a treatment for psoriatic arthritis (PsA) and other immune-mediated conditions such as psoriasis. Objectives: To assess the involvement of IL-17F in chronic inflammation in tissue from patients with PsA and disease-relevant cells, and to determine the effect of dual neutralisation of IL-17A and IL-17F in suppressing inflammation, compared with blockade of IL-17A.

Methods: Synovial and lesional skin tissue from patients with PsA was probed by immunostaining for expression of IL-17F protein. Normal dermal fibroblasts and synoviocytes, in the presence of TNF $\alpha$, were stimulated with recombinant IL-17A and IL-17F to assess the inflammatory response. Using cytokine-specific blocking antibodies, the individual and combined effects of IL-17A and IL-17F were explored with: pro-inflammatory cytokine expression in a complex in vitro model (synoviocytes from patients with PsA and normal dermal fibroblasts were treated with pro-inflammatory mediators from supernatant [SN] of sorted Th17 cells), microarray and cell migration studies.

Results: IL-17F expression was observed in tissue biopsies from patients with PsA. In normal dermal fibroblasts, normal synoviocytes and synoviocytes from patients with PsA, stimulation with recombinant IL-17F promoted production of pro-inflammatory mediators, such as IL-6 and IL-8, though to a lesser extent than with recombinant IL-17A. Treatment of Th17 SN-stimulated synoviocytes from patients with PsA with bimekizumab (neutralising IL-17A and IL-17F) led to greater reductions of IL-6 $(42 \%$ lower $p<0.05)$ and IL-8 $(35 \%$ lower $\mathrm{p}<0.05)$ production than IL-17A inhibition. Bimekizumab treatment of Th17 SN-stimulated normal dermal fibroblasts also reduced production of IL-6 (35\% lower $p<0.0001)$ and IL-8 $(57 \%$ lower $\mathrm{p}<0.0001)$ more than IL-17A alone. Combining IL-17A + IL-17F monoclonal antibodies produced similar results to bimekizumab. Levels of expression of 27 inflammation-linked genes, including CXCL1, CXCL2, CXCL3 and IL-15RA, were lower with dual neutralisation of IL-17A and IL-17F by bimekizumab versus inhibition of IL-17A. Suppression of migration of neutrophils (Fig.) and monocytes, both involved in tissue destruction in immune-mediated diseases, was substantially greater with bimekizumab treatment than with single blockade of IL-17A.

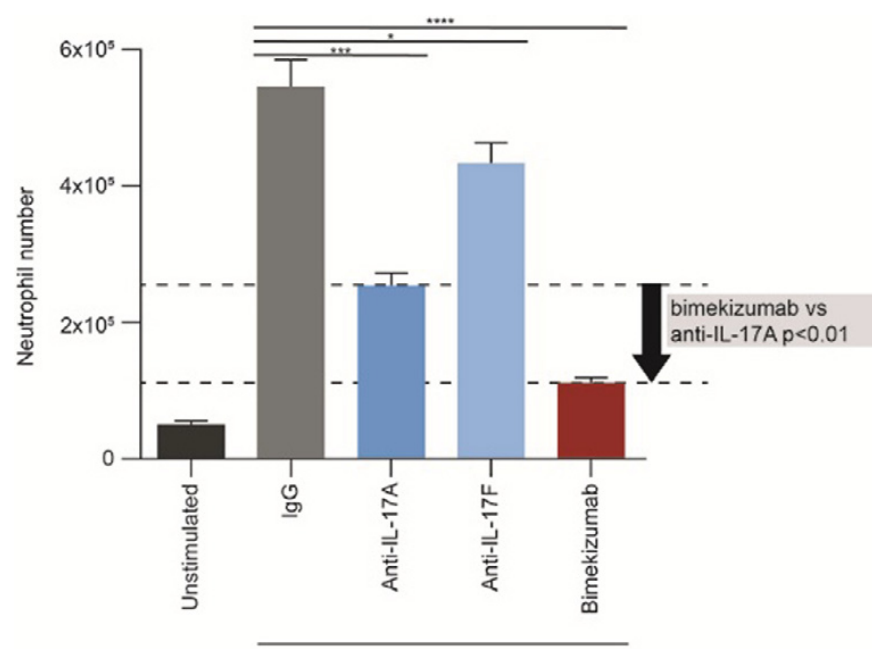

Th17 supernatant

Fig. Quantification of neutrophil migration following treatment with cytokine-specific antibodies and bimekizumab. "represents a significant reduction of cell migration vs $\lg G$ control, $" p<0.05, "{ }^{\prime} p<0.01, \cdots p<0.001, \cdots " \cdots<0.0001$

Conclusions: Dual neutralisation of IL-17A and IL-17F provides evidence for the contribution of IL-17F to inflammation in joints and skin beyond IL-17A alone. As a result, dual inhibition of IL-17A and IL-17F by bimekizumab may provide an effective treatment for immune-mediated inflammatory diseases such as PsA.

References:

[1] C Johansen et al. Br J Dermatol 2009:160:319-24.

[2] AL Lima et al. Br J Dermatol 2016:174:514-21.

[3] C Doe et al. Chest 2010:138:1140-7.

Disclosure of Interest: A. Maroof Employee of: UCB Pharma, R. Okoye Employee of: UCB Pharma, T. Smallie Employee of: UCB Pharma, D. Baeten Grant/research support from: UCB Pharma, Consultant for: AbbVie, Pfizer, MSD, Roche, BMS, Novartis, Eli Lilly, Boehringer Ingelhaim and Glenmark, S. Archer Employee of: UCB Pharma, C. Simpson Employee of: UCB Pharma, M. Griffiths Consultant for: UCB Pharma, Employee of: UCB Pharma, S. Shaw Employee of: UCB Pharma DOI: 10.1136/annrheumdis-2017-eular.4966

\section{THU0039 LIPIDOMICS ANALYSIS OF HDL PARTICLE IN INFLAMMATORY RHEUMATIC DISEASES: ALTERATION OF PHOSPHOLIPID COMPOSITION AND ROLE OF INFLAMMATION}

C. Giraud ${ }^{1}$, F. Capel ${ }^{2}$, F. Dutheil ${ }^{3}$, B. Pereira ${ }^{4}$, M. Soubrier ${ }^{1}$, A. Kontush ${ }^{5}$, M. Lhomme ${ }^{6}$, J.L. Sebedio ${ }^{2}$, A. Tournadre ${ }^{1} .{ }^{1}$ Rheumatology, CHU Gabriel Montpied; ${ }^{2}$ Unité nutrition humaine, UMR 1019/Inra; ${ }^{3}$ AME2P, Blaise Pascal University; ${ }^{4}$ Biostatistic/ "Delegation Recherche Clinique et Innovation", CHU Gabriel Montpied, Clermont Ferrand; ${ }^{5}$ INSERM UMR_s 1166 - Ican, Université Pierre et Marie Curie - Paris 6; ${ }^{6}$ ICANalytics, ICAN, Paris, France

Background: Rheumatoid arthritis (RA), psoriatic arthritis (PsA) and ankylsosing 
spondylitis (SA) are associated with an increased cardiovascular (CV) mortality. Quantitative abnormalities in lipid profiles are insufficient to explain this excess of $\mathrm{CV}$ risk and a qualitative approach of HDL composition is required to identify loss of atheroprotective functions and to correctly identify patients at risk. Atheroprotective functions of $\mathrm{HDL}$ are directly linked to the structure of $\mathrm{HDL}$ mainly composed of phospholipids (PL).

Objectives: The main objective of this study is to analyze the PL composition of $\mathrm{HDL}$ in patients with chronic inflammatory rheumatic diseases and to compare to matched healthy controls.

Methods: HDL structure was assessed in patients with active RA (ACR criteria), PSA (CASPAR criteria) and SA (ASAS criteria) patients before initiating first biologic and in healthy controls matched for age, sex and body mass index. Dyslipidemia treatment or pathology which could interfere with lipid profile were excluded. Demographics data, disease activity, cardiometabolic profile and plasma samples were collected. HDL particle were isolated from plasma after two step ultracentrifugation using gradient of density. Lipidomics analysis were performed using liquid chromatography coupled with mass spectrometry. Phospholipid composition between patients and controls was compared using multivariate analyses to take into account possible confounding variables determined according to univariate results and clinical relevance (age, tobacco consumption, steroids use). Multidimensional analyses as factorial mixed data analysis (FMDA) were performed to complete these analyses.

Results: 19 RA, 19 PsA and 12 SA were analyzed (table 1). 220 phospholipids species were identified among which 2 major classes were modified in rheumatic diseases. Phosphatidylcholine (PC) decreased in RA and PsA ( $p<0.01$ and $<0.05$ respectively) while lysophosphatidylcholine (LPC) increased significantly $(p<0.01$, and $<0.05$ respectively). Some phospholipids species as PC 40: $8(p<0.001)$, LPC 16:0 $(p<0.001)$, LPC 18:0 $(p<0.001)$ were identified as discriminant marker of HDL composition in rheumatic disease as compared to controls.

\begin{tabular}{|c|c|c|c|c|c|c|}
\hline & \begin{tabular}{|l|}
$R A$ \\
(meansso)
\end{tabular} & $\begin{array}{l}\text { Controls } \\
\text { (meantiv) }\end{array}$ & \begin{tabular}{|l} 
PSA \\
(meent id)
\end{tabular} & $\begin{array}{l}\text { Controls } \\
\text { (memntso) }\end{array}$ & \begin{tabular}{|l|} 
SA \\
(meantsda)
\end{tabular} & $\begin{array}{l}\text { Controls } \\
\text { (meantude) }\end{array}$ \\
\hline Number of pasients & 19 & 18 & 19 & 17 & 12 & 12 \\
\hline Ase & $55.32 \pm 10,91$ & $58.78 \pm 4.61$ & $49.63 \div 8.04 *$ & $57.65 \pm 4.77 \cdot \cdot$ & $45.33 \pm 6.56 \cdots$ & $57.17 \pm 5.69 \cdots$ \\
\hline Sese $(W / M)(B S M)$ & $1 / 28(5.3 \times)$ & $1 / 17(5,5 \times)$ & 1099(536) & $10 /(58 \times)$ & $8 / 4(67 \times)$ & $8 / 4(67 \times)$ \\
\hline OMI (ned/m') & $24.22 \pm 4 ., 81$ & $22.13 * 2,92$ & 27.7887 .60 & $24.55+3.63$ & 25.4994.16 & 23.691292 \\
\hline APOB/APOAL1 & $0.67 \div 0,15$ & $0.63 \pm 0,15$ & $0.77 \div 0.21$ & $0.75 \pm 0.14$ & $0.74 \pm 0.12$ & $0.75+0.17$ \\
\hline CRP $(m e / 2)$ & $31.16 \pm 43.98 \cdots$ & $0.80 \pm 0,72 \cdots$ & $19.74 \div 17.33 \cdots$ & $2.102183 \cdots$ & $288 \div 1.97^{\circ}$ & $1912.17 \%$ \\
\hline CV family history $\mathrm{Y} / \mathrm{N}(\mathrm{X})$ & |2/18 (5.3\%) & O/18 (0N) & $1 / 18(5,250)$ & o/17106) & $0 / 12(0 x)$ & o/12(100) \\
\hline CV perional nstory Y/N $(8)$ & a/19(0) (1) & $0 / 18(0 x)$ & o/19(0) & $0 / 17(105)$ & $0 / 12(0 \infty)$ & $0 / 12(05)$ \\
\hline Mich blood pressure $Y / N(X)$ & $4 / 15(21 \times)^{*}$ & $0 / 18(0 \times))^{*}$ & $2 / 17(111 \times)$ & $0 / 17(0 \times)$ & $4 / 8(3))^{*} \cdot$ & $0 / 12$ (105). \\
\hline Disbetes mellitus $\mathrm{Y} / \mathrm{N}(\mathrm{X})$ & 1/18 (5.3\%) & o/18 (0N) & $1 / 18(5,25)$ & $0 / 17(105)$ & (0/12(10N) & o/12(10x) \\
\hline Current tabacco use $Y / N(x)$ & $5 / 12(265))^{*}$ & $0 / 18(0 \times))^{*}$ & $5 / 24(26 \times)$ & $0 / 11(0 \times)$ & $6 / 6$ (5OS) $\cdot *$ & $0 / 12(00 x) \cdot \cdot$ \\
\hline Disease duration (vears) & $6,68856,33$ & & & & & \\
\hline DAS 28 CRP score & $4.5611,05$ & & 4.0020 .99 & & & \\
\hline BASOAN & & & $55.67 \pm 1858$ & & 61.14414.09 & \\
\hline Corticosteroil treatment $Y / N(X)$ & |10/9 (533) & & $2 / 17(110)$ & & $1 / 11(8,30)$ & \\
\hline NSW treatment $Y / N(X)$ & $9 / 10(47 \times)$ & & $6 / 13(325)$ & & 6/6 (50\%) & \\
\hline Methotrexate treatment $\mathrm{Y} / \mathrm{N}(\mathrm{S})$ & 15/4 (796) & & 9/10(47) & & $1 / 11(8,36)$ & \\
\hline
\end{tabular}

Conclusions: Phospholipids composition of HDL is altered in RA and PsA. These alterations could explain a loss of atheroprotective functions and the excess of CV risk observed in RA and PsA patients. Chronic inflammation, through the activation of phospholipase A2 type II which hydrolyze PC into LPC, could modify the structure of HDL phospholipids and thus could impact HDL functionality such as cholesterol efflux, LDL oxidation modulation, anti-inflammatory and vasculoprotective properties. These preliminary data suggest the major role of inflammation in these alterations.

\section{References:}

[1] Tselepis AD et al. Atheroscler Suppl. 2002;3(4):57-68.

[2] Kontush A, et al. J Lipid Res. 2013;54(11):2950-63.

Disclosure of Interest: None declared

DOI: 10.1136/annrheumdis-2017-eular.5491

\section{THU0040 NOVEL AKT ACTIVATOR SC-79 IS A POTENTIAL TREATMENT FOR ALCOHOL-INDUCED OSTEONECROSIS OF THE FEMORAL HEAD}

C. Yixuan. Orthopedics, Shanghai Jiao Tong University Affiliated Sixth People's Hospital, Shanghai, China

Background: Alcohol is known to be one ofthe leading risk factors for osteonecrosis of the femoral head. However, the underlying etiology and protective strategiesof alcohol-induced osteonecrosis of the femoral headhavenot been clarified.

Objectives: The aim of this study was to explore the molecular mechanism of alcohol-induced osteonecrosis of the femoral head, and to investigate the protective effect of SC-79 on the disease.

Methods: In vitro, we employed RT-PCR, alizarin red staining, alkaline phosphatase activity testing, western blot, immunofluorescence staining to investigate the effect of ethanol on hBMSCs. In vivo experiments, immunofluorescence staining, TRAP, TUNEL and micro-CT were performed to investigate the development of ONFH.

Results: In vitro, we found that ethanol could significantly impair the expression of osteogenic genes of RUNX2 and OCN, downregulate osteogenic differentiation, impair IGF-1 induced membrane recruitment of the Akt, suppress the Akt-Ser473 phosphorylationand the subsequent activation of Akt/GSK3 $\beta / \beta$-catenin signaling in bone mesenchymal stem cells. Functional studies further confirmed this signaling was the critical mediator during the ethanol-induced inhibitory effects on osteogenesis of BMSCs. Thus, the dephosphorylation of Akt-Ser473 in Akt/GSK3 $\beta / \beta$-catenin signaling pathway might be a potential mechanism in the pathogenesis of alcoholinduced osteonecrosis of the femoral head.SC-79, a novel Akt activator was introduced in this study to block the dephosphorylation effect of ethanol on AktSer473 both in vitro and in vivo. In the rat model of alcohol-induced osteonecrosis of the femoral head, micro-CT and histopathological analyses revealed obvious osteonecrosis changes in alcohol-administrated rats while significantly less developed in SC-79 injected rats. OPN, OCN and COL1 immunofluorescence staining revealed that osteogenic response of femoral heads was markedly reduced after alcohol administration, but significantly reversed by SC-79 treatment.
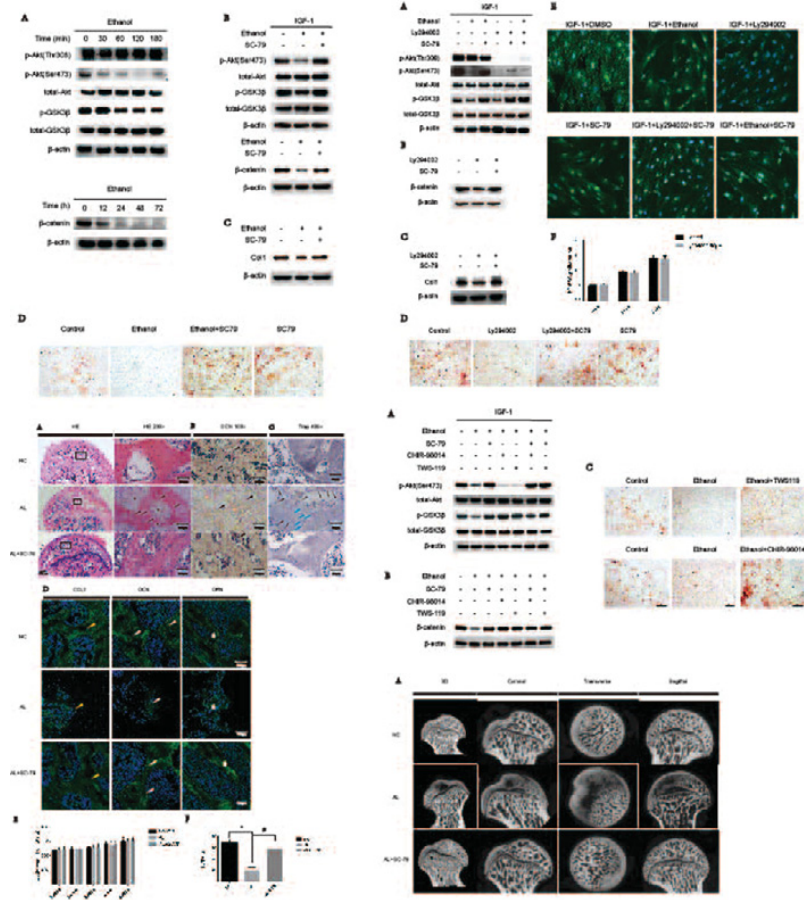

Conclusions: Hence, we discovered alcohol-induced osteonecrosis of the femoral

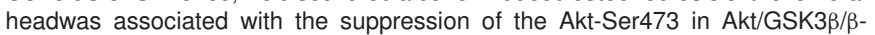
catenin pathway in BMSCs. The administration of SC-79, to elevate Akt activation, might be a clinical strategy to prevent the development of alcoholinducedosteonecrosis of the femoral head.

Acknowledgements: The current study was supported by National Natural Science Foundation of China $(81272003,81301572)$ and SMC-Chen Xing Plan for Splendid Young Investigators of Shanghai Jiao Tong University.

Disclosure of Interest: None declared

DOI: 10.1136/annrheumdis-2017-eular.1927

\section{THU0041 PROGRANULIN AND DERIVATIVES CAN BE EMPLOYED AS LATENT DUAL-FUNCTION CHONDROGENIC AND ANTI-INFLAMMATORY THERAPEUTICS FOR THE TREATMENT OF RHEUMATOID ARTHRITIS}

C.M.A. Schultz, Y. Chernajovsky. Bone \& Joint Research Unit, Queen Mary, University of London, London, United Kingdom

Background: Rheumatoid Arthritis (RA) is a debilitating inflammatory disease of the joints afflicting around $1 \%$ of Western populations. Some of the best treatments are anti-TNF agents, but these only achieve remission in $60 \%$ of patients and cause deleterious side effects. Progranulin (PGRN), a cysteine-rich, multi-domain growth factor was reported to bind TNF-receptors (TNFR) blocking pro-inflammatory signalling ${ }^{2}$, as well as stimulating chondrogenesis ${ }^{3}$. PGRN is cleavable and its peptides have pleiotropic effects, some of which may be beneficial and others refractory to ameliorating RA. Granulin A (GRN A) was shown to interact with cartilage ECM protein COMP ${ }^{4}$. Atsttrin - comprising 3 fused PGRN regions, was shown to ameliorate arthritic disease ${ }^{2}$. The latency associated peptide (LAP) of TGF $\beta 1$ can be fused to short peptides and cytokines via a MMP cleavage site to facilitate targeting to inflamed sites such as RA joints, reducing side effects and enhancing in vivo half-life ${ }^{1}$. We hypothesised that a peptide based on PGRN could be fused to LAP and used to both block TNF and stimulate cartilage regeneration in RA joints in a targeted way.

Objectives: To produce a panel of PGRN derivatives fused to LAP. To determine the chondrogenic and anti-TNF capacities of the fragments in the presence and absence of MMP activation. To evaluate their efficacy in the CIA model of RA. 